\title{
A Parallel Tabu Search Algorithm for Optimizing Multiobjective VLSI Placement
}

\author{
Mahmood R. Minhas and Sadiq M. Sait \\ College of Computer Sciences \& Engineering, \\ King Fahd University of Petroleum \& Minerals, \\ Dhahran-31261, Saudi Arabia \\ \{minhas, sadiq\}@ccse.kfupm.edu.sa
}

\begin{abstract}
In this paper, we present a parallel tabu search (TS) algorithm for efficient optimization of a constrained multiobjective VLSI standard cell placement problem. The primary purpose is to accelerate TS algorithm to reach near optimal placement solutions for large circuits. The proposed technique employs a candidate list partitioning strategy based on distribution of mutually disjoint set of moves among the slave processes. The implementation is carried out on a dedicated cluster of workstations. Experimental results using ISCAS-85/89 benchmark circuits illustrating quality and speedup trends are presented. A comparison of the obtained results is made with the results of a parallel genetic algorithm (GA) implementation.
\end{abstract}

\section{Introduction and Related Work}

General iterative heuristics such as tabu search and genetic algorithms (GAs) have been widely used to solve numerous hard problems [1. This interest is attributed to their generality, ease of implementation, and ability to reach near optimal solutions by escaping from local minima. However, depending on size of a problem, such heuristics may have huge runtime requirements. This is also true for VLSI placement problem of modern industry-size circuits for which, iterative heuristics require huge run times to reach near optimal solutions [2,3]. With rapidly increasing density of VLSI circuits, the run time dilemma of iterative techniques is aggravating and hence there is a need of accelerating their search process.

One way to adapt iterative techniques to large problems and to efficient traversing of large search space is their parallelization [4,5]. An effective parallelization strategy must consider issues such as proper partitioning of the problem to facilitate uniform distribution of computationally intensive tasks. At the same time, it should be capable of thorough traversal of the complex search space. In the subsequent paragraphs, we present a brief review of some earlier efforts towards parallelization of TS.

A number of parallelization techniques have been reported in literature [6]. A taxonomy of parallel tabu search strategies was given by Crainic et. al [7. In the most straightforward and widely adopted approach, $k$ tabu search processes are spawned and run concurrently on $k$ processors where each processor 
carries out independent search 6, 8. Malek et al. suggested linking independent searches where each slave runs a copy of a serial TS but with different parameter settings [9]. After specified time intervals, all the slave processes are halted and the master process selects the overall best solution found so far and broadcasts it to all the slave processes. Each slave process then restarts its search process from the best solution it receives from the master.

Another proposed approach is to parallelize search process within an iteration of TS. In this approach, each process is given the task of exploring a subset of the neighborhood of the current solution. Here two approaches are followed: synchronous and asynchronous. In the synchronous approach, various processes are always working with the same solution but exploring different partitions of the neighborhood. The master process orchestrates the activities of the slave processes [8]. In the asynchronous approach, all processes are peer and are usually not working with the same current solution 6. Both of these approaches require that the set of possible moves be partitioned among the available processors so that each processor can explore a distinct sub-region of the neighborhood.

Some other suggestions for efficient parallelization of TS include partitioning the search space, or partitioning the problem into smaller sub-problems with determining the best moves for each sub-problem, and then performing a compound move [6].

Attempts to solve some hard optimization problems have also been reported in literature. Parallel tabu search algorithms for the vehicle routing problem are presented in [8,10]. A massively parallel implementation of tabu search for the Quadratic Assignment Problem (QAP) is reported in 11. The parallel algorithm was implemented on a Connection Machine CM-2 (a massively parallel SIMD machine). A reduction in runtime per iteration was reported when compared to some other sequential and parallel implementations [11,12].

In this paper, we present a parallel TS algorithm for efficient optimization of a hard optimization problem namely, constrained multiobjective VLSI standard cell placement. The rest of the paper is organized as follows: The next section briefly discusses the placement problem and the related cost functions. In Section 3. some implementation details of the proposed parallel tabu search algorithm are presented, followed by experimental results and comparisons in Section 4. Section 5 presents some concluding remarks.

\section{The Placement Problem and Cost Functions}

We address a multiobjective VLSI standard cell placement problem where the objectives are optimization of and total wirelength, power consumption, and timing performance (delay), whereas layout width is considered as a constraint. Cell placement is one of the intermediate steps in VLSI physical design and is a generalization of QAP.

The VLSI cell placement problem can be stated as follows [13: Given a collection of cells or modules with ports (inputs, outputs, power and ground pins) on the boundaries, the dimensions of these cells (height, width, etc), and a collection 


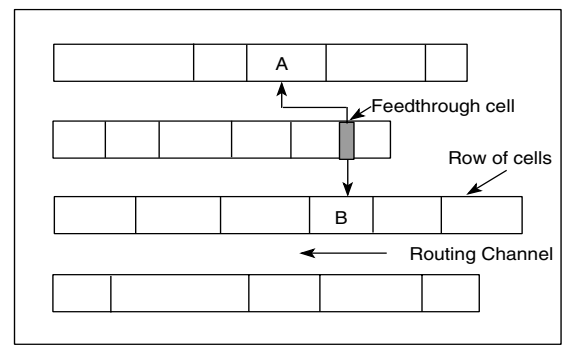

Fig. 1. A Typical Standard Cell Layout

of nets (which are sets of ports that are to be wired together), placement problem consists of finding suitable physical locations for each cell on the layout. By suitable we mean those locations that minimize given objective functions, subject to some constraints imposed by the designer, the implementation process, or layout strategy and the design style.

In this work, we deal with standard cell placement, where all the circuit cells are constrained to have the same height, while the width of the cell is variable and depends upon its complexity. A typical standard cell layout is shown in Figure 1. As can be seen in the figure, cells are arranged in rows with routing channels between the rows. Due to varying width of cells, row width may be unequal depending on the type and number of cells placed in a row. An approximation would be to treat cells as points, but in order to have a more accurate estimate of wirelength, widths of cells are taken into account. Heights of routing channels are estimated using the vertical constraint graphs constructed during the channel routing phase. With this information, a fairly accurate estimate of power dissipation, delay and total wirelength can be obtained [13]. Next, we formulate the cost function used in our optimization process.

\subsection{Cost Functions}

Now we formulate cost functions for our three objectives and for the width constraint.

Wirelength Cost: Interconnect wirelength of each net in the circuit is estimated using steiner tree approximation. Total wirelength is computed by adding all these individual estimates:

$$
\text { Cost }_{\text {wire }}=\sum_{i \in M} l_{i}
$$

where $l_{i}$ is the wirelength estimation for net $i$ and $M$ denotes total number of nets in circuit.

Power Cost: Power consumption $p_{i}$ of a net $i$ in a circuit can be given as:

$$
p_{i} \simeq C_{i} \cdot S_{i}
$$


where $C_{i}$ is total capacitance of net $i$, and $S_{i}$ is the switching probability of net $i$. Here, $C_{i}$ depends on wirelength of net $i$, hence Equation 2 can be written as:

$$
p_{i} \simeq l_{i} \cdot S_{i}
$$

The cost function for total power consumption in the circuit can be given as:

$$
\text { Cost }_{\text {power }}=\sum_{i \in M} p_{i}=\sum_{i \in M}\left(l_{i} \cdot S_{i}\right)
$$

Delay Cost: The delay cost is determined by computing delay along the longest path in a circuit. The delay of any given path is computed as summation of the delays of all the nets belonging to the path and the switching delays of the cells driving these nets. Hence, the delay $T_{\pi}$ of a path $\pi$ consisting of nets $\{1,2, \ldots, k\}$ is expressed as:

$$
T_{\pi}=\sum_{i=1}^{k-1}\left(C D_{i}+I D_{i}\right)
$$

where $C D_{i}$ is the switching delay of the cell driving net $i$ and $I D_{i}$ is the interconnect delay of net $i$. The placement phase affects $I D_{i}$ only because $C D_{i}$ is technology dependent and is hence independent of the placement process. The cost function for delay of the circuit can be given as:

$$
\operatorname{Cost}_{\text {delay }}=\max \left\{T_{\pi}\right\}
$$

Width Cost: Width cost is given by the maximum of all the row widths in the layout. We have constrained layout width not to exceed a certain positive ratio $\alpha$ to the average row width $w_{\text {avg }}$, where $w_{\text {avg }}$ is the minimum possible layout width obtained by dividing the total width of all the cells in the layout by the number of rows in the layout. We can express width constraint as below:

$$
\text { Width }-w_{\text {avg }} \leq \alpha \times w_{\text {avg }}
$$

\section{$2.2 \quad$ Fuzzification of Multiobjectives}

Since we are targeting to optimize three (possibly conflicting) objectives simultaneously, we need to formulate an aggregating cost function which can expresses the costs of the objectives in form of a single quantity. We resorted to using fuzzy in designing our aggregating cost function. Fuzzy logic allows to describe the objectives in terms of linguistic variables following which, the membership functions can be defined. The membership functions for SMALL wirelength, LOW power consumption, and SHORT delay are same as described in [3. Finally, fuzzy rules are used to design the aggregate cost function. In this work, we have used the following fuzzy rule:

Rule 1: IF a solution has $S M A L L$ wirelength AND $L O W$ power consumption AND SHORT delay THEN it is an GOOD solution. 
The above rule is translated to and-like OWA fuzzy operator [14] and the membership $\mu(x)$ of a solution $x$ in fuzzy set GOOD solution is obtained by:

$$
\mu(x)= \begin{cases}\beta \cdot \min _{j=l, p, d}\left\{\mu_{j}(x)\right\}+(1-\beta) \cdot \frac{1}{3} \sum_{j=l, p, d} \mu_{j}(x) & \text { if } W i d t h-w_{\text {avg }} \leq \alpha \cdot w_{\text {avg }} \\ 0 ; & \text { otherwise. }\end{cases}
$$

Here $\mu_{j}(x)$ for $j=\{l, p, d\}$ are the membership values in the fuzzy sets SMALL wirelength, LOW power consumption, and SHORT delay respectively, whereas $\beta$ is a constant in range $[0,1]$. A placement solution that results in a higher value of $\mu(x)$ is considered a better solution.

\section{Parallel Tabu Search Algorithm for Optimizing VLSI Placement}

The parallel tabu search strategy adopted in this work was engineered after a careful performance analysis of our sequential TS implementation [3]. The analysis was performed using some profiling tools (like GNU profiler) to obtain insight into determining the time consuming operations of the code and the usage of resources. For the circuits experimented on, it was found that almost $60 \%$ to $80 \%$ of the total run time was spent on cost computation of the three objectives and their fuzzification. Furthermore, experiments with parameters revealed that for our hard optimization problem with conflicting multiobjectives, large sizes of candidate list (upto 120) were required to obtain high quality solutions. Since cost computation for all moves in the candidate list was the most time consuming operation, the proposed algorithm was designed with a view of partitioning this compute-intensive task. The proposed algorithm employs a candidate list partitioning strategy based on distribution of mutually disjoint set of moves among the slave processes. The pseudo code of the master and the slave processes in the proposed parallel TS are shown in Figures 2 and 3 respectively.

According to taxonomy given by Crainic et. al [7, our approach can be classified as a synchronous master-slave (one master and remaining slaves), 1control (each process is responsible for its search), Rigid Synchronous (RS) (all processes are forced to establish communication and exchange information at specific points) and Single Point Single Strategy (SPSS) (all the processes start with the same initial solution and follow the same strategy).

In this implementation, there is an initialization step during which, the master process generates and sends an initial solution and a disjoint (non-overlapping) partial candidate list (PCL) to each slave process. A move in a PCL assigned to a slave in a particular iteration does not appear in PCLs assigned to other slaves. Each slave process searches its local neighborhood by trying each move in the partial candidate list on the initial solution and computes gains due to them. Then it sends the best move and its corresponding cost (or gain) to the 


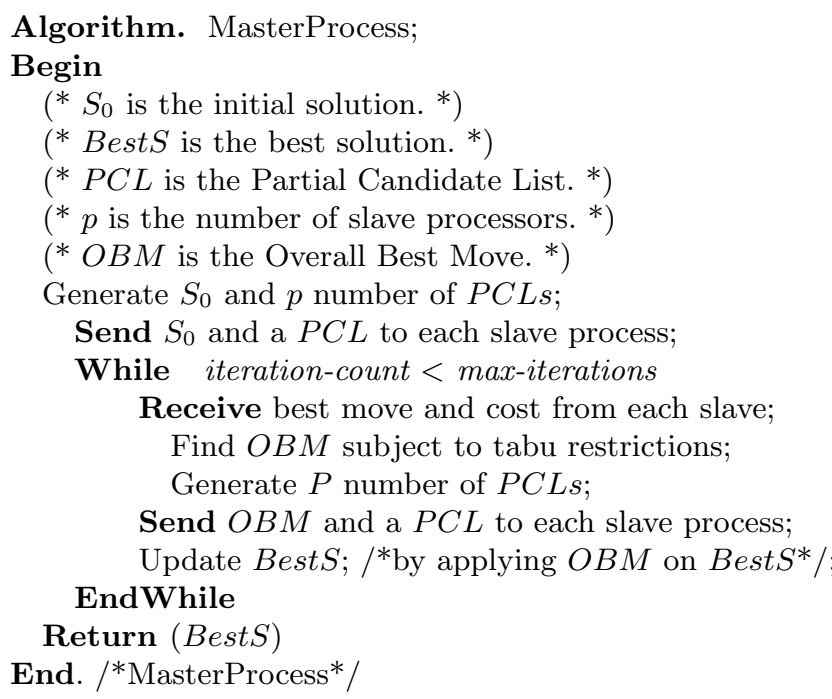

Fig. 2. The master process in parallel TS

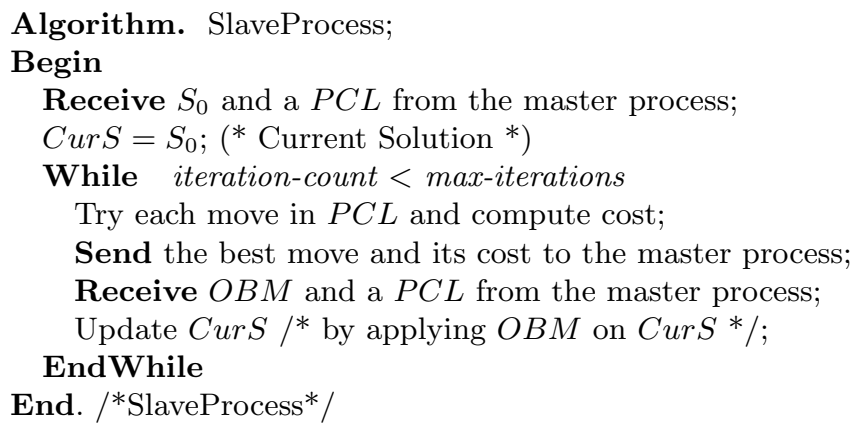

Fig. 3. The slave process in parallel TS

master process. The master process selects the overall best move (OBM) among the moves it received from slave processes subject to tabu restrictions. Then in each subsequent iteration, the master process sends the overall best move and a new partial candidate list to each slave process. Each slave process now starts by performing the received overall best move so that all the slave processes start their iteration from the same solution. Each slave process searches its local neighborhood and sends the best move and its cost to the master process.

\section{Experimental Results and Discussions}

The experimental setup consists of the a homogeneous cluster of 8 machines (where 1 machine is always working as a master processor), x86 architecture, Pentium- 4 of $2 \mathrm{GHz}$ clock speed, and $256 \mathrm{MB}$ of memory. These machines are 
connected by $100 \mathrm{Mbit} / \mathrm{s}$ Ethernet switch. Operating system used in RedHat Linux 7.3 (kernel 2.4.7-10). The paradigm used for parallelization is MPI (Message Passing Interface). Specifically, MPICH (a portable implementation of MPI standard 1.1) is used in our implementation. In terms of GFlops measure, the maximum performance of the cluster, with NAS Parallel Benchmarks was found to be 1.6 GFlops, (using NAS's LU, Class A, for 8 processors). Using this same benchmark for a single processor, the individual performance of one machine was found out to be 0.3 GFlops. The maximum bandwidth that was achieved using PMB was $91.12 \mathrm{Mbits} / \mathrm{sec}$, with an average latency of $68.69 \mu$ sec per message. ISCAS-85/89 circuits are used as performance benchmarks for evaluating the proposed parallel TS placement technique. These circuits are of various sizes in terms of number of cells and paths, and thus offer a variety of test cases.

For comparison purposes, we also implemented a parallel genetic algorithm (GA) which is a derivative of a standard distributed GA and follows the island model, with independently evolving sub-populations and periodic exchanges of solutions through migration [15,16]. A pseudo-diversity approach is taken, wherein similar solutions are not permitted in the population at any time. This diversity serves to widen the search, while limiting the possibility of premature convergence in local minima solution space. The initial population is constructed at the master process and distributed among $N$ slave processes which start running serial GA on their allocated population for a predefined number of iterations called the Migration Frequency $(M F)$. Then each slave process sends $M R$ (Migration Rate) number of its best solutions to the master process, which selects $M R$ overall best solutions and broadcasts them to all slave processes. Each slave process absorbs the incoming best solutions into its population (if they are not already found) by replacing the weakest solutions. Each slave process then continues with the serial GA for another $M F$ iterations. Standard PMX crossover is used to generate offsprings [1].

The quality of solution obtained and run time required using different number of processors for both TS and GA are tabulated in Table1, For each circuit, the number of cells are given in the table. The ' $\mu(s)$ TS' and ' $\mu(s)$ GA' columns show the aggregate fuzzy membership of solution obtained by TS and by GA respectively, whereas ' $p$ ' denotes the number of processors used. It should be noted that run times shown are for achieving a certain fixed quality.

In case of large circuits, parallel GA was unable to find a reasonable quality solution even after running for a large amount of time. Even for smaller circuits, the solution quality obtained using TS is significantly superior to that obtained using GA, and also the speedup trend is very consistent for TS. On the other hand, parallel GA did not show such performance or trend.

The proposed parallel TS has shown a consistent trend in terms of speedup with increasing number of processors. Figure 4 shows a run-time as well as a speedup plot of parallel TS for some selected large circuits, and demonstrates an almost linear speedup. As can be seen, there is a consistent decreasing trend in run time. This trend is more pronounced for medium to large circuits than for smaller ones and reveals the good scalability of the proposed approach. 
Table 1. Run times and solution quality $\mu(s)$ for achieving a target membership for serial and parallel TS/GA approaches. X indicates unreasonably high run time requirement

\begin{tabular}{|c|c|c|c|c|c|c|c|c|c|c|c|c|c|c|}
\hline \multirow{2}{*}{$\begin{array}{l}\text { Circuit } \\
\text { Name }\end{array}$} & \multirow{2}{*}{$\begin{array}{l}\text { Number } \\
\text { of Cells }\end{array}$} & \multirow{2}{*}{$\begin{array}{c}\mu(s) \\
\mathrm{TS}\end{array}$} & \multirow{2}{*}{\begin{tabular}{|c|} 
Time for \\
Serial TS
\end{tabular}} & \multicolumn{6}{|c|}{ Time for Parallel TS } & \multirow{2}{*}{$\begin{array}{c}\mu(s) \\
\mathrm{GA}\end{array}$} & \multirow{2}{*}{$\begin{array}{l}\text { Time for } \\
\text { Serial GA }\end{array}$} & \multicolumn{3}{|c|}{ Time for Parallel GA } \\
\hline & & & & $\mathrm{p}=2$ & $\mathrm{p}=3$ & $\mathrm{p}=4$ & $\mathrm{p}=5$ & $\mathrm{p}=6$ & $\mathrm{p}=7$ & & & $\mathrm{p}=3$ & $\mathrm{p}=5$ & $\mathrm{p}=7$ \\
\hline s386 & 172 & 0.688 & 52 & 28 & 20 & 17 & 16 & 15 & 14 & 0.504 & 15 & 9.9 & 5.7 & 6.7 \\
\hline s641 & 433 & 0.785 & 934 & 472 & 332 & 239 & 205 & 171 & 151 & 0.616 & 793 & 307 & 390 & 289 \\
\hline $\mathrm{s} 832$ & 310 & 0.644 & 74 & 40 & 33 & 23 & 22 & 20 & 19 & 0.479 & 128 & 43 & 37 & 39 \\
\hline s953 & 440 & 0.661 & 195 & 98 & 71 & 53 & 46 & 41 & 36 & 0.511 & 309 & 136 & 91 & 108 \\
\hline s1196 & 561 & 0.653 & 374 & 187 & 132 & 97 & 88 & 78 & 67 & 0.484 & 988 & 327 & 262 & 205 \\
\hline s1488 & 667 & 0.603 & 259 & 131 & 93 & 69 & 63 & 55 & 49 & 0.482 & 1883 & 677 & 435 & 418 \\
\hline s1494 & 661 & 0.601 & 268 & 137 & 96 & 72 & 65 & 57 & 51 & 0.496 & 1405 & 847 & 638 & 479 \\
\hline c3540 & 1753 & 0.665 & 2142 & 1146 & 703 & 547 & 440 & 370 & 344 & - & $\mathrm{X}$ & $\mathrm{X}$ & $\mathrm{X}$ & $\mathrm{X}$ \\
\hline s3330 & 1961 & 0.699 & 1186 & 590 & 451 & 313 & 245 & 210 & 184 & - & $\mathrm{X}$ & $\mathrm{X}$ & $\mathrm{X}$ & $\mathrm{X}$ \\
\hline c5378 & 2993 & 0.669 & 1850 & 914 & 601 & 467 & 371 & 312 & 264 & - & $\mathrm{X}$ & $\mathrm{X}$ & $\mathrm{X}$ & $\mathrm{X}$ \\
\hline s9234 & 5844 & 0.631 & 5571 & 2855 & 2006 & 1525 & 1272 & 1062 & 849 & - & $\mathrm{X}$ & $\mathrm{X}$ & $\mathrm{X}$ & $\mathrm{X}$ \\
\hline
\end{tabular}

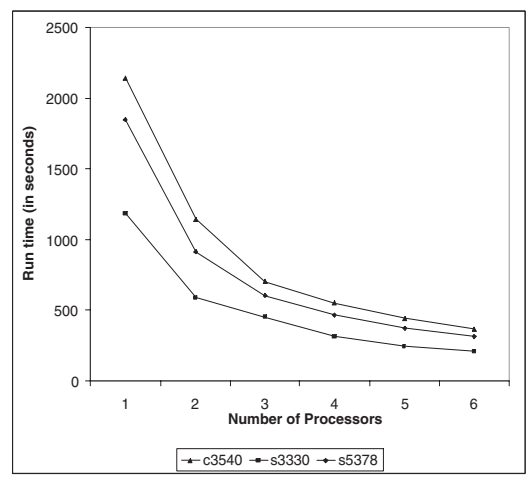

(a)

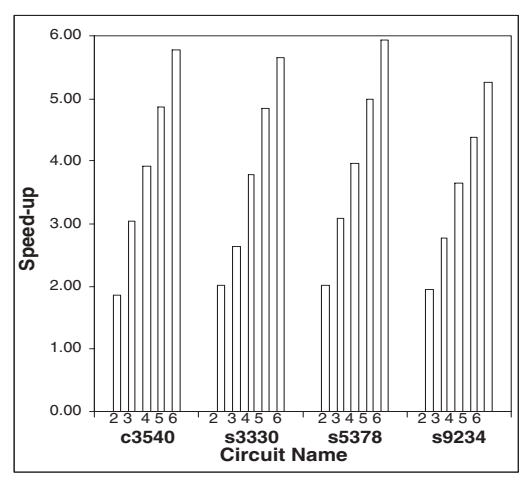

(b)

Fig. 4. (a) Decrease in run times for selected large circuits with increasing number of processors for parallel TS. (b) Speedup obtained for selected large circuits for parallel TS

\section{Conclusions}

In this work, we presented a parallel tabu search strategy for accelerating the solution to a constrained multiobjective VLSI cell placement problem. The proposed strategy belongs to p-control, RS, MPSS class and was implemented on a dedicated cluster of workstations. A distributed parallel GA was also implemented for the comparison purposes. Experimental results on ISCAS-85/89 benchmarks exhibit that the proposed parallel TS shows an excellent trend in terms of speedup and requires far lesser run times as compared to serial TS for obtaining the same quality of placement solutions. When compared with results obtained by parallel GA, the proposed parallel TS clearly outperforms both in terms of solution quality as well as run time.

\section{Acknowledgment}

The authors would like to thank King Fahd University of Petroleum \& Minerals, Dhahran, Saudi Arabia, for support under project code \# COE/CELLPLACE/263. 


\section{References}

1. Sadiq M. Sait and Habib Youssef. Iterative Computer Algorithms and their Application to Engineering. IEEE Computer Society Press, December 1999.

2. Sadiq M. Sait, Habib Youssef, Aiman El-Maleh, and Mahmood R. Minhas. Iterative Heuristics for Multiobjective VLSI Standard Cell Placement. Proceedings of IJCNN'01, International Joint Conference on Neural Networks, 3:2224-2229, July 2001.

3. Sadiq M. Sait, Mahmood R. Minhas, and Junaid A. Khan. Performance and LowPower Driven VLSI Standard Cell Placement using Tabu Search. Proceedings of the 2002 Congress on Evolutionary Computation, 1:372-377, May 2002.

4. Prithviraj Banerjee. Parallel Algorithms for VLSI Computer-Aided Design. Prentice Hall International, 1994.

5. Van-Dat Cung, Simone L. Martins, Celso C. Riberio, and Catherine Roucairol. Strategies for the Parallel Implementation of metaheuristics. Essays and Surveys in Metaheuristics, pages 263-308, Kluwer 2001.

6. I. De Falco, R. Del Balio, E. Tarantino, and R. Vaccaro. Improving Search by Incorporating Evolution Principles in Parallel Tabu Search. In Proc. of the first IEEE Conference on Evolutionary Computation- CEC'94, volume 1, pages 823828, June 1994.

7. T. G. Crainic, M. Toulouse, and M. Gendreau. Towards a Taxonomy of Parallel Tabu Search Heuristics. INFORMS Journal of Computing, 9(1):61-72, 1997.

8. Bruno-Laurent Garica, Jean-Yves Potvin, and Jean-Marc Rousseau. A Parallel Implementation of the Tabu Search Heuristic for Vehicle Routing Problems with Time Window Constraints. Computers $\&$ Operations Research, 21(9):1025-1033, November 1994.

9. M. Malek, M. Guruswamy, M. Pandya, and H. Owens. Serial and parallel Simulated Annealing and Tabu Search algorithms for the Traveling Salesman Problem. Annals of Ops. Res., 21:59-84, 1989.

10. E. Taillard. Parallel Iterative Search Methods for the Vehicle Routing Problem. Networks, 23:661-673, 1993.

11. J. Chakrapani and J. Skorin-Kapov. Massively Parallel Tabu Search for the Quadratic Assignment Problem. Annals of Operations Research, 41:327-341, 1993.

12. E. Taillard. Robust Tabu Search for the Quadratic Assignment Problem. Parallel Computing, 17:443-455, 1991.

13. Sadiq M. Sait and Habib Youssef. VLSI Physical Design Automation: Theory and Practice. World Scientific, Singapore, 2001.

14. Ronald R. Yager. On Ordered Weighted Averaging Aggregation Operators in Multicriteria Decision Making. IEEE Transaction on Systems, MAN, and Cybernetics, 18(1), January 1988.

15. M. Toulouse, T. G. Crainic, and M. Gendreau. Issues in Designing Parallel and Distributed Search Algorithms for Discrete Optimization Problems. Publication CRT96-36, Centre de recherche sur les transports, Université de Montréal, Montréal, Canada, 1996.

16. Erick Cant-Paz. A Survey of Parallel Genetic Algorithms. Calculateurs Parallles, Reseaux et Systems Repartis, 1998. 